\title{
Radiologische Bildgebungsbefunde bei Brustsarkomen
}

\section{Radiological Imaging Findings in Breast Sarcomas}

\section{Einleitung}

Brustsarkome stellen eine seltene und aggressive Tumorentität dar, die weniger als $1 \%$ aller malignen Neoplasien der Brust betrifft [Adem C et al. Br J Cancer 2004; 91: 237-241]. Sie treten überwiegend bei Frauen auf, das männliche Geschlecht ist nur mit 1,5\% betroffen [Matsumoto RAEK et al. Radiol Bras 2018; 51: 401-406]. Die jährliche Inzidenz beträgt ca. 4,6 Fälle/ 1000000 Frauen. Das mittlere Erkrankungsalter liegt bei ca. 40-50 Jahren. Es zeigt sich eine ausgeglichene Seitenverteilung; ein bilateraler Brustbefall ist selten. Brustsarkome bilden histologisch eine sehr heterogene Gruppe unterschiedlicher Tumorsubtypen. Des Weiteren unterscheiden wir primäre von sekundären Brustsarkomen, wobei bei beiden das Angiosarkom die häufigste Tumorentität darstellt. Dieses geht entweder als Hämangiosarkom von entdifferenzierten Endothelien der Blutgefäße oder als Lymphangiosarkom von Endothelien der Lymphgefäße hervor.

Die genaue Pathogenese und Ätiologie der primären Brustsarkome sind bislang unklar. Prädisponierende Faktoren sind u. a. Genmutationen wie das Li-FraumeniSyndrom und die Neurofibromatose Typ I. Des Weiteren spielen umweltassoziierte Faktoren wie beispielsweise Arsen-haltige Verbindungen, Herbizide und Immunsuppressiva eine Rolle [Adem C et al. Br J Cancer 2004; 91: 237-241]. Primäre Brustsarkome präsentieren sich klinisch meist als schmerzlose, mobile und unilaterale Brustschwellung, die im Gegensatz zu den Karzinomen nicht an der Haut und Muskelfaszie fixiert sind und durch ein schnelleres Wachstum und Tumordurchmesser im Median zwischen 5-6 cm auffallen [Yang WT et al. Radiology 2007; 242: 725-734]. Sekundäre Brustsarkome können nach einer Radiatio nach brusterhaltender Therapie eines Mammakarzinoms oder aber aufgrund eines chronischen Lymphödems nach radikaler Mastektomie auftreten. Das klinische Erscheinungsbild reicht von bläulich-violetten bis erythematösen Hautveränderungen bis hin zu knotig-
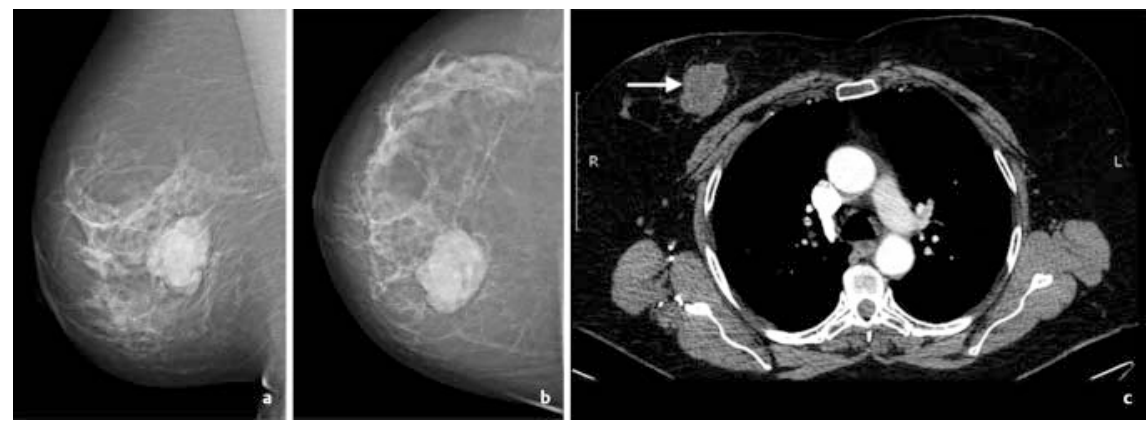

- Abb. 1 a 69-jährige Frau mit einem Tastbefund der rechten Brust. Die Mammografie in a mlo- und $\mathbf{b}$ cc-Projektion zeigt einen hyperdensen, ovalär konfigurierten und unscharf begrenzten ca. $6 \mathrm{~cm}$ messenden Herdbefund im unteren inneren Quadranten rechts. c In der Computertomografie (CT) des Thorax in axialer Schichtführung nach Kontrastmittelapplikation weist der Herdbefund eine irreguläre, randständig betonte Kontrastmittelaufnahme auf (Pfeil). Kein Nachweis von axillären oder pulmonalen Metastasen (nicht abgebildet). Histologie: Fibrosarkom.
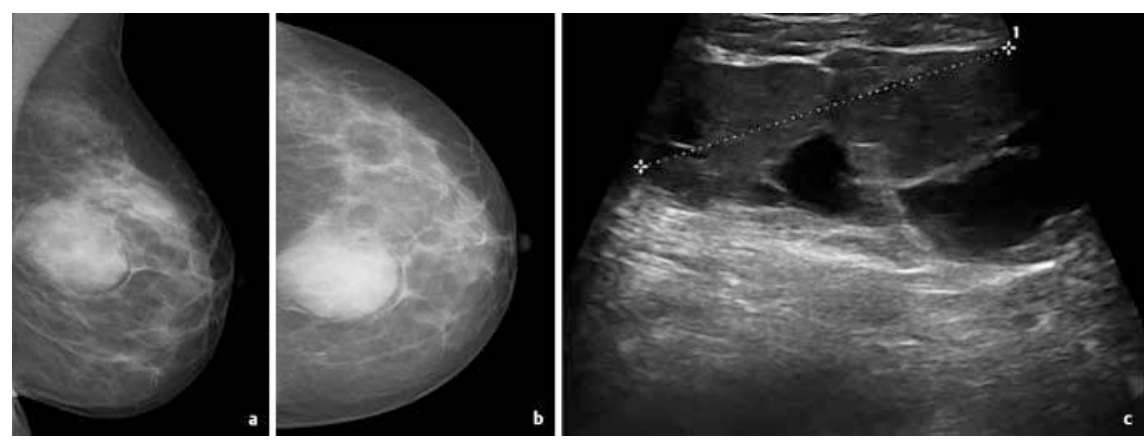

- Abb. 2 a 42-jährige Frau mit einem Tastbefund der linken Brust. Die Mammografie in a mlound b cc-Projektion zeigt einen hyperdensen, ovalär konfigurierten, unscharf begrenzten Herdbefund bei ca. 12 Uhr im hinteren Parenchymdrittel der linken Brust. c In der Sonografie findet sich ein ovalär konfigurierter, unscharf begrenzter, heterogener Herdbefund mit einer Ausdehnung von ca. 3 cm. Histologie: Fibrosarkom.

polypoiden Tumoren. Diese Veränderungen sind im Gegensatz zu den primären Brustsarkomen auf die Kutis und Subkutis begrenzt.

Die hämatogene Metastasierung bei Brustsarkomen mit Beteiligung von Lungen, Knochenmark und Leber sowie deutlich seltener der Haut, Milz, Thorax, Gehirn und retroperitoneal steht in deutlichem Gegensatz zu der sonst häufigen lymphatischen Metastasierung von Mammakarzinomen. Der Lymphknotenstatus spielt somit bei Brustsarkomen eine untergeordnete Rolle, da lymphatische Tumorabsiedlungen mit ca. $10 \%$ äußert selten auftreten [Mat- sumoto RAEK et al. Radiol Bras 2018; 51 : 401-406].

\section{Fallbeschreibung}

Die radiologischen Charakteristika konzentrieren sich auf Fallserien zwischen 5 und 42 Fällen [Surov A et al. Acta Radiol 2011; 52: 597-601; Wienbeck $S$ et al. Eur J Radiol 2017; 88: 1-7]. Die Abklärungsdiagnostik sollte in gleicher Art und Weise wie bei allen Brustbefunden erfolgen. Die radiologischen Befunde allein lassen keine eindeutige Differenzierung in primäre und sekundäre Brustsarkome und ihre Subtypen zu. 

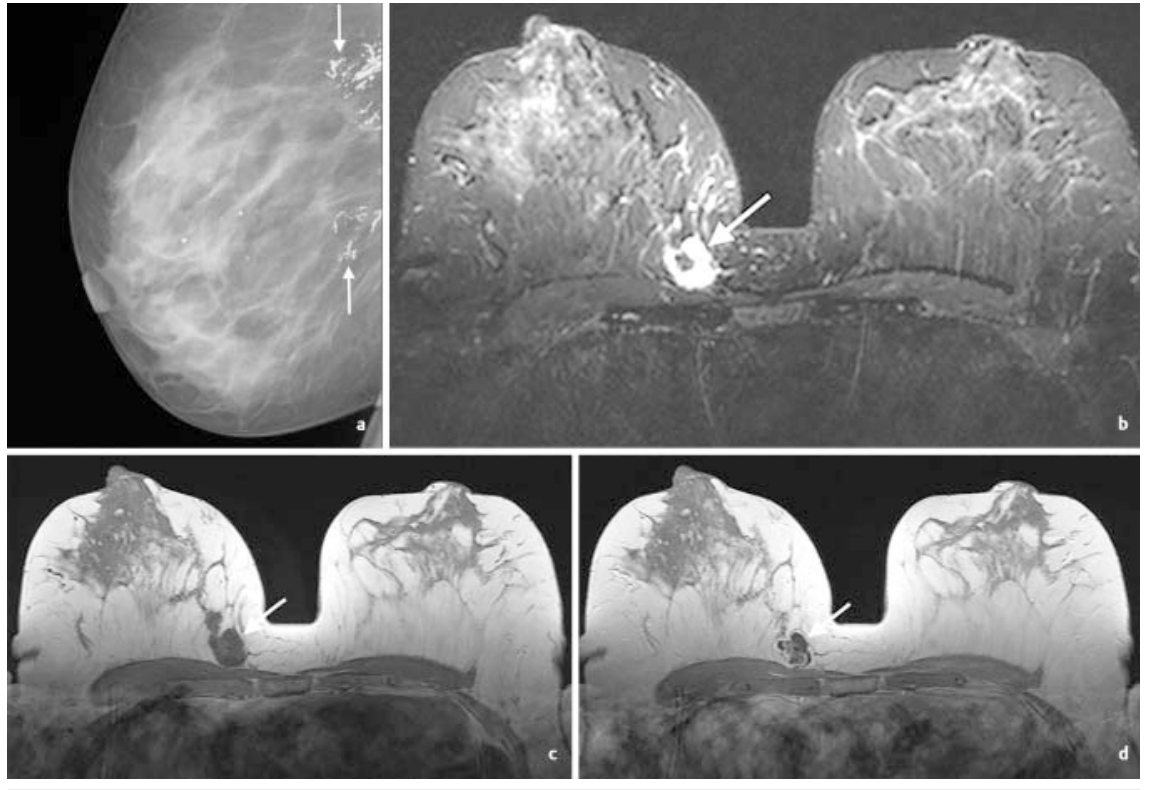

- Abb.3 a 63-jährige Frau mit einem schmerzlosen Tastbefund der rechten Brust. a In der Mammografie in Im-Projektion zeigen sich gruppiert angeordnete grobschollige Verkalkungen im hinteren Parenchymdrittel der rechten Brust (Pfeile). Des Weiteren sind benigne Mikro- und Makroverkalkungen im Sinne von verkalkten Zysten im zentralen Drüsenkörper abgrenzbar. b Die Magnetresonanztomografie (MRT) in axialer Schichtführung zeigt einen ovalär konfigurierten, unscharf begrenzten Herdbefund mit hyperintensem Signalverhalten in der short-tau-inversion-recovery (STIR) -Sequenz (Pfeil). c In der nativen T1-gewichteten Sequenz zeigt dieser ein hypointenses Signalverhalten (Pfeil). d In der T1-gewichteten Sequenz nach Kontrastmittelapplikation ergibt sich eine intensive, randständige Kontrastmittelaufnahme mit zentraler Nekrosezone. Histologie: myxoides Chondrosarkom.

Mammografisch finden sich als Befundcharakteristika Herdbefunde und Architekturstörungen, wobei erstgenannte mit ca. $90 \%$ am häufigsten auftreten. Herdbefunde zeigen hierbei eine irreguläre oder ovale Form mit unscharfer Randbegrenzung [Wienbeck S et al. Eur] Radiol 2017; 88: 1-7] ( Abb. 1a, b, 2). Die Befundcharakteristika spikulierte Ränder und Verkalkungen werden hingegen nur sehr selten beschrieben ( $\mathbf{A} \mathbf{b} \mathbf{b}$. 3a). Das Auftreten von fokalen Asymmetrien begrenzt sich auf Einzelfallberichte [Yang WT et al. Radiology 2007; 242: 725-734] ( Abb. 4).

Sonografisch zeigen sich die Befunde überwiegend hypoechogen, irregulär konfiguriert, mit unscharfer Begrenzung und dorsaler Schallabschwächung [Wienbeck S et al. Eur J Radiol 2017; 88: 1-7] ( $\triangleright$ Abb. 2c). Hautverdickungen oder auffällige axilläre Lymphknoten fehlen in der Regel beim primären Brustsarkom. Hingegen wird das sekundäre postradiogene Angiosarkom überwiegend durch entsprechende Hautveränderungen der Thoraxwand diagnostiziert.
In der Magnetresonanztomografie (MRT) zeigen sich die Befunde heterogen hypointens in der T1-gewichteten Sequenz, hyperintens in der T2-gewichteten Sequenz und weisen nach Kontrastmittelapplikation überwiegend eine Typ-2-Kinetik auf [Wienbeck S et al. Eur] Radiol 2017; 88: 1-7] (• Abb. 3b-d, 5, 6).

Die Bildgebung mittels Computertomografie (CT) wird häufig im Rahmen des Stagings angewendet. Die Befunde weisen eine irreguläre Randbegrenzung und randständig betonte Kontrastmittelaufnahme auf ( $\triangleright$ Abb. 1c).

Als mögliche Differenzialdiagnosen müssen große juvenile Fibroadenome und ein Phylloidestumor in Betracht gezogen werden, die im Vergleich zu den primären Brustsarkomen eine ähnliche klinische Symptomatik und vergleichbare Befundcharakteristika in der Mammografie und Sonografie zeigen ( $\triangleright$ Abb. 7). Des Weiteren sind primäre Brustsarkome von einem triple-negativen Brustkarzinom und metaplastischen Karzinom zu differenzieren, wobei letztgenannte Tumorentität häufiger mit einem Befall der axillären Lymph-

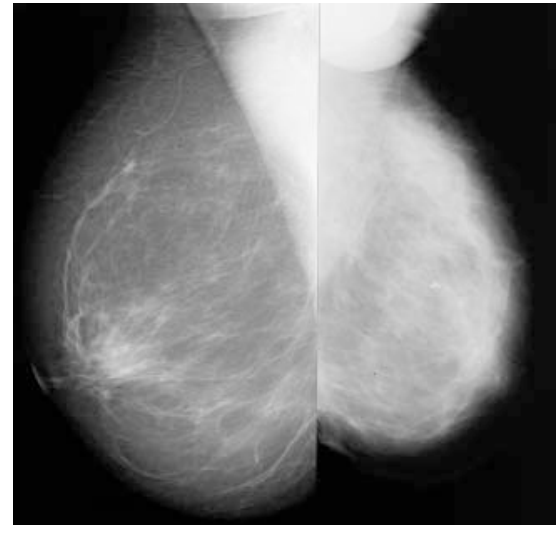

- Abb.4 62-jährige Frau mit einem schmerzlosen Tastbefund der linken Brust. Die Mammografie in mlo-Projektion beider Brüste zeigt eine Zunahme der Drüsengewebsdichte im oberen Quadranten der linken Brust als Ausdruck einer fokalen Asymmetrie. Anlagebedingt besteht eine Anisomastie der linken Brust. Histologie: Angiosarkom.

knoten einhergeht [Matsumoto RAEK et al. Radiol Bras 2018; 51: 401-406].

Therapeutisch und prognostisch stellt bei Brustsarkomen die chirurgische R0Resektion die Behandlung der Wahl dar. Als lokale Therapiemaßnahme ist eine radikale Mastektomie mit negativen Tumorrändern anzustreben. Eine Resektion der axillären Lymphknoten wird nicht routinemäßig empfohlen, es sei denn, eine Lymphknotenmetastasierung wurde bestätigt. Zur Anwendung einer Chemound Radiotherapie bei Sarkomen der Brust besteht kein eindeutiger Konsens und wird derzeit bei hochgradigen oder großen Tumoren empfohlen.

\section{Diskussion}

Brustsarkome weisen keine eindeutigen pathognomonischen radiologischen Veränderungen auf, sodass eine sichere Differenzierung in primäre und sekundäre Brustsarkome sowie ihre einzelnen Tumorsubtypen bildgebend nicht sicher möglich ist. Bei ihrem Auftreten muss neben benignen Befunden ebenfalls an ein invasives Mammakarzinom gedacht werden. Die Befunde präsentieren sich mammografisch überwiegend als nichtkalzifizierte, irregulär oder oval konfigurierte Herdbefunde und deutlich seltener als Architekturstörungen. In der Sonografie sind die 

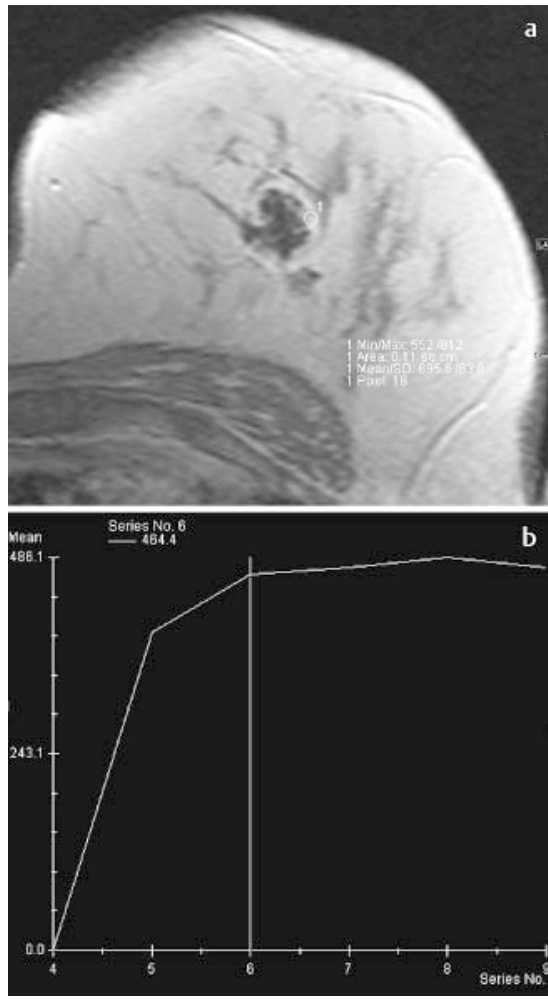

- Abb. 5 a 58-jährige Frau mit einem Tastbefund der linken Brust. a Die Magnetresonanztomografie (MRT) in axialer Schichtführung zeigt einen ovalär konfigurierten, unscharf begrenzten Herdbefund mit intensiver, randständig betonter Kontrastmittelaufnahme und Nekrosezone in der T1-gewichteten Sequenz nach Kontrastmittelapplikation. b Die kinetische Analyse zeigt einen raschen Signalanstieg $>100 \%$ und eine anschließende Plateauphase nach Kontrastmittelapplikation (Kurventyp 2). Histologie: Leiomyosarkom, G2.

meisten Befunde hypoechogen, irregulär konfiguriert und zeigen eine unscharfe Begrenzung mit dorsaler Schallabschwächung. Die Kontrastmittelkinetik in der MRT nach i.v. KM-Gabe ergibt überwiegend einen Kurventyp 2. Eine anamnestische und klinische Korrelation ist daher bei einer schnellen Wachstumstendenz und einem einseitigen Befallsmuster zusammen mit den entsprechenden radiologischen Befunden zwingend erforderlich. Die Kenntnis der radiologischen Befunde ersetzt jedoch nicht die zügige minimalinvasive Abklärungsdiagnostik mittels Biopsie. Das Risiko einer Zellverschleppung durch den Stichkanal ist prinzipiell gegeben. Therapeutisch spielt die chirurgische R0-Resektion mit entsprechend großem Sicherheitsabstand, überwiegend mittels
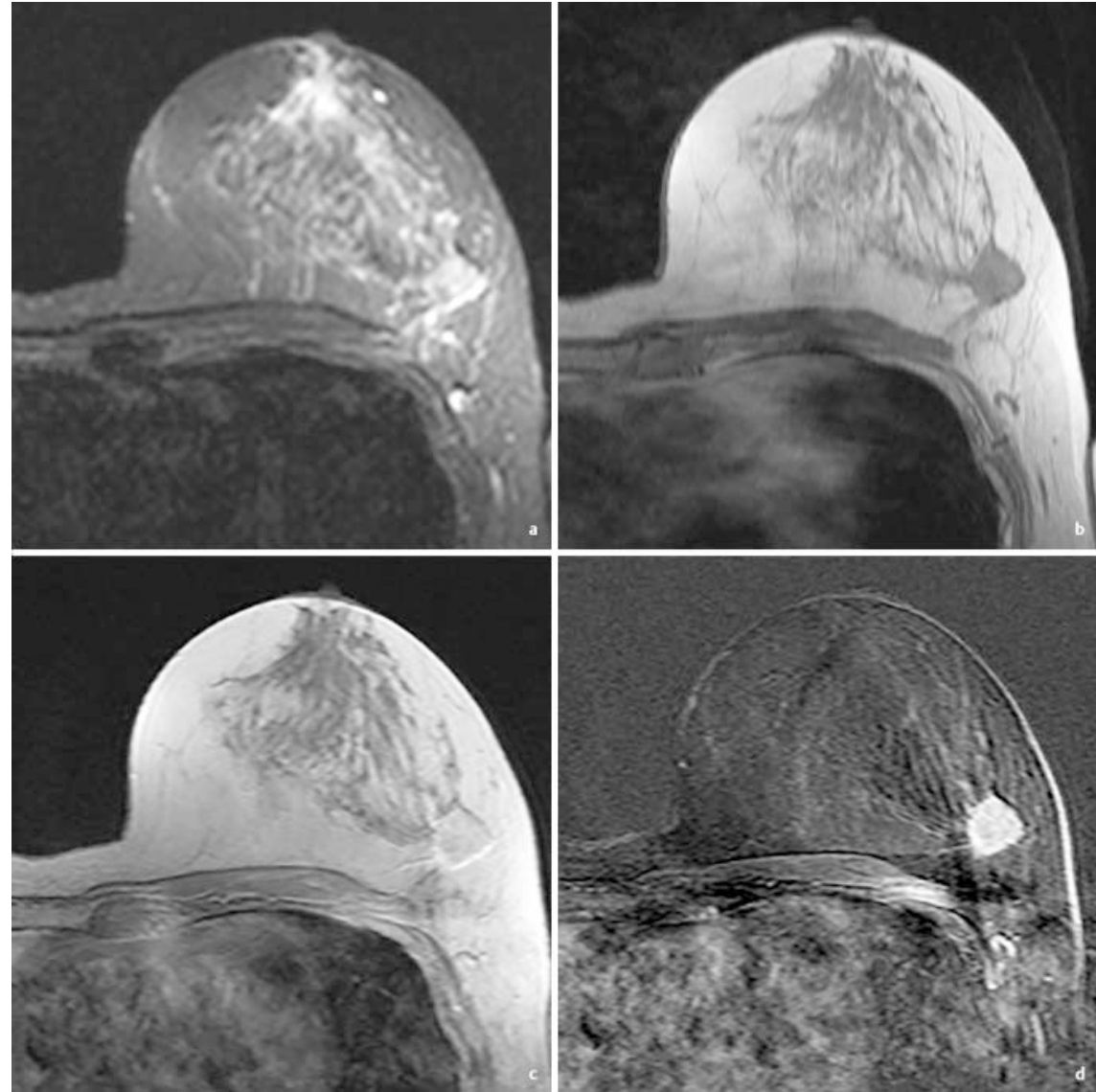

- Abb. 6 a Magnetresonanztomografie (MRT) in axialer Schichtführung der linken Brust bei einer 58-jährigen Frau. a Die short-tau-inversion-recovery (STIR) -Sequenz zeigt einen runden, unscharf begrenzten Herdbefund mit hyperintensem Signalverhalten. b In der nativen T1-gewichteten Sequenz findet sich ein hypointenses Signalverhalten. c Dieser zeigt eine homogene Kontrastmittelaufnahme in der T1-gewichteten Sequenz nach Kontrastmittelapplikation und d in der 2. Subtraktionsphase. Histologie: Angiosarkom.

Mastektomie, die prognostisch wichtigste Rolle.

\section{Schlussfolgerung}

Die möglichen radiologischen Veränderungen sollten zusammen mit der entsprechenden Klinik und Anamnese an ein Brustsarkom denken lassen. Diese ersetzen jedoch nicht die zügig einzuleitenden weiteren Abklärungsmaßnahmen mittels minimalinvasiver Biopsie.

\section{KERNAUSSAGEN}

- Brustsarkome weisen keine einheitlichen Befunde in der radiologischen Bildgebung auf.

- Brustsarkome können gutartige Befunde nachahmen.
- Ein zügiges minimalinvasives Vorgehen mittels Biopsie ist erforderlich.

Interessenkonflikt

Die Autoren geben an, dass kein Interessenkonflikt besteht.

\section{Autorinnen/Autoren}

Susanne Wienbeck ${ }^{1}$, Hans Jonas Meyer ${ }^{2}$, Alexey Surov ${ }^{2}$

${ }^{1}$ Institute for Diagnostic and Interventional Radiology, University Medical Center Göttingen, Göttingen, Germany

${ }^{2}$ Department for Diagnostic and Interventional Radiology, University-Hospital Leipzig, Germany 

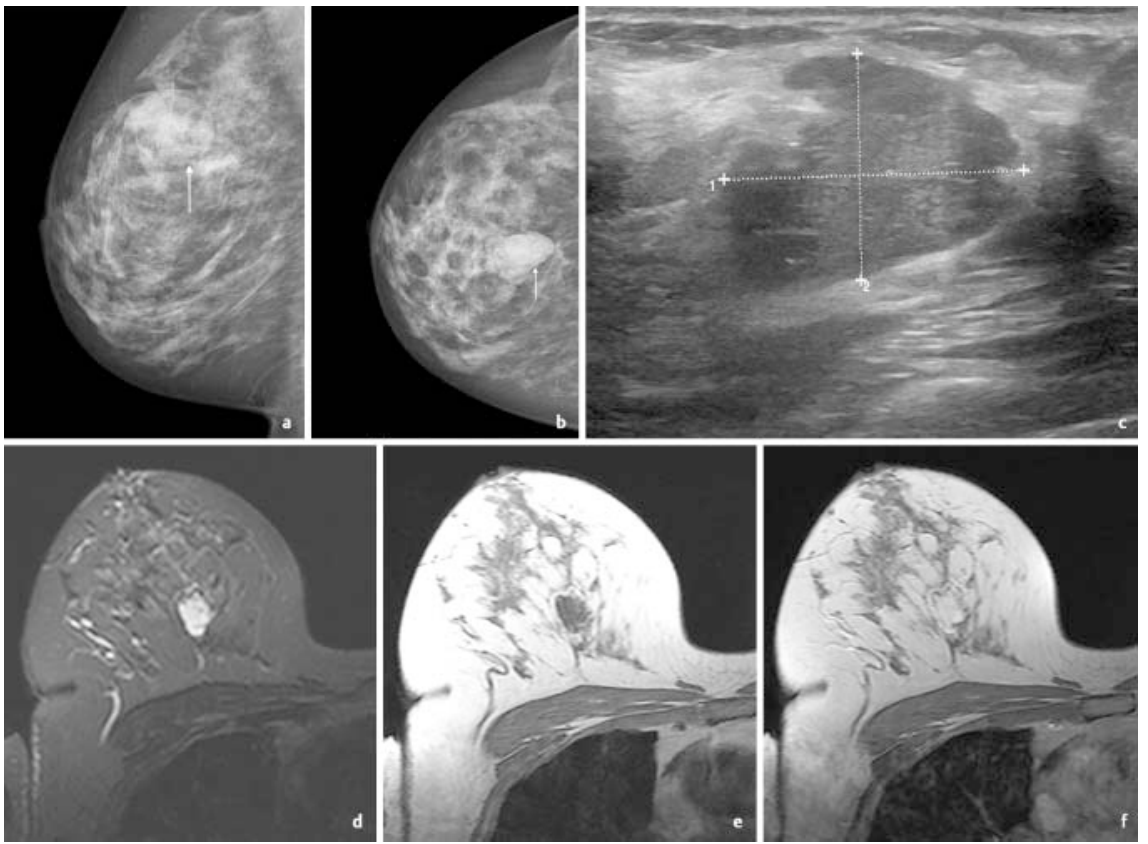

Abb. 7 a 64-jährige Frau mit einem Tastbefund der rechten Brust. a Die Mammografie in mlo-Projektion und $\mathbf{b}$ cc-Projektion zeigen einen hyperdensen, ovalär konfigurierten und unscharf begrenzten Herdbefund bei 12 Uhr rechts (Pfeil). Ebenfalls findet sich abgrenzbarer disseminiert verteilter benigner Mikrokalk. c Die Sonografie ergibt einen ovalär konfigurierten, unscharf begrenzten, heterogenen Herdbefund mit einer Ausdehnung von ca. $3 \mathrm{~cm}$. d Der Herdbefund zeigt in der Magnetresonanztomografie (MRT) in axialer Schichtführung ein hyperintenses Signalverhalten in der short-tau-inversion-recovery (STIR) -Sequenz. e Dieser stellt sich hypointens in der nativen T1-gewichteten Sequenz dar. $\mathbf{f}$ In der T1-gewichteten Sequenz nach Kontrastmittelapplikation findet sich eine intensive, homogene Kontrastmittelaufnahme. Histologie: Phylloidestumor.
Korrespondenzadresse

\section{Dr. Susanne Wienbeck}

Institute for Diagnostic and Interventional

Radiology, Universitätsklinikum Göttingen

Robert-Koch-Str. 40

37075 Göttingen

Germany

Tel.: ++49/05 51/3968965

Fax: ++49/0551/3969606

susanne.wienbeck@med.uni-goettingen.de

\section{Bibliografie}

DOI https://doi.org/10.1055/a-0993-5418

Online-Publikation: 28.8.2019

Fortschr Röntgenstr 2020; 192: 219-222

(c) Georg Thieme Verlag KG, Stuttgart · New York ISSN 1438-9029 